\title{
Spatial variation and socio-economic determinants of Plasmodium falciparum infection in northeastern Tanzania
}

\author{
Bruno P Mmbando ${ }^{1 *}$, Mathias L Kamugisha', John P Lusingu' ${ }^{1}$, Filbert Francis ${ }^{1}$, Deus S Ishengoma', \\ Thor G Theander ${ }^{2}$, Martha M Lemnge ${ }^{1}$ and Thomas H Scheike ${ }^{3}$
}

\begin{abstract}
Background: Malaria due to Plasmodium falciparum is the leading cause of morbidity and mortality in Tanzania. According to health statistics, malaria accounts for about 30\% and 15\% of hospital admissions and deaths, respectively. The risk of $P$. falciparum infection varies across the country. This study describes the spatial variation and socio-economic determinants of $P$. falciparum infection in northeastern Tanzania.

Methods: The study was conducted in 14 villages located in highland, lowland and urban areas of Korogwe district. Four cross-sectional malaria surveys involving individuals aged 0-19 years were conducted during short (Nov-Dec) and long (May-Jun) rainy seasons from November 2005 to June 2007. Household socio-economic status (SES) data were collected between Jan-April 2006 and household's geographical positions were collected using hand-held geographical positioning system (GPS) unit. The effects of risk factors were determined using generalized estimating equation and spatial risk of $P$. falciparum infection was modelled using a kernel (nonparametric) method.
\end{abstract}

Results: There was a significant spatial variation of $P$. falciparum infection, and urban areas were at lower risk. Adjusting for covariates, high risk of $P$. falciparum infection was identified in rural areas of lowland and highland. Bed net coverage levels were independently associated with reduced risk of $P$. falciparum by $19.1 \%$ (95\%Cl: 8.9$28.2, p<0.001$ ) and by $39.3 \%$ (95\%Cl: 28.9-48.2, $p<0.001$ ) in households with low and high coverage, respectively, compared to those without bed nets. Households with moderate and lower SES had risk of infection higher than $60 \%$ compared to those with higher SES; while inhabitants of houses built of mud walls were at 15.5\% (95\%Cl: 0.1 - 33.3, $p<0.048$ ) higher risk compared to those living in houses built by bricks. Individuals in houses with thatched roof had an excess risk of $17.3 \%(95 \% \mathrm{Cl}: 4.1-32.2, \mathrm{p}<0.009)$ compared to those living in houses roofed with iron sheet.

Conclusions: There was high spatial variation of risk of $P$. falciparum infection and urban area was at the lowest risk. High bed net coverage, better SES and good housing were among the important risk factors associated with low risk of $P$. falciparum infection.

\section{Background}

Malaria due to Plasmodium falciparum is a major public health problem in Sub-Saharan Africa and accounts for about $90 \%$ of malaria disease burden in the world. It has been estimated to cost approximately $\$ 12$ billion per year due to the devastating effects of the disease and it affects

\footnotetext{
* Correspondence: bmmbando@tanga.mimcom.net

${ }^{1}$ National Institute for Medical Research, Tanga Centre, Tanzania

Full list of author information is available at the end of the article
}

the social and economic development of the region [1]. In Tanzania, malaria is the leading cause of morbidity and mortality and it accounts for about $30 \%$ and $15 \%$ of hospital admissions and deaths, respectively [2].

Malaria transmission intensity varies with season and decreases with altitude due to its dependence on temperature which affects the development of vectors and parasites [3]. High level of urbanization is also associated with less malaria and this is attributable to high

\section{Biomed Central}


pollution levels which affects mosquito larvae development, good housing [4], better access to health facilities and high bed nets coverage $[5,6]$. Other factors which affects transmission of malaria include rainfall, topography, land use and socio-economic status (SES) [7]. Changes in natural environment has also been associated with changing malaria transmission [7].

Application of geographical information system (GIS) and spatial statistical methods are regarded as important tools in epidemiology to identify areas with increased risk of diseases and determine spatial association between disease and risk factors [8-11]. In studies of distribution of malaria for example, it is not possible to measure and display all the variables that are associated with malaria transmission in a given geographical area. The use of maps and spatial statistical methods makes it easy to identify and display the unmeasured effect as a spatial effect and show areas with unusual high rates of the disease. Thus, disease-specific maps play an important role in disease control activities including monitoring the changes of the disease epidemiology, guiding resource allocation as well as identifying areas for further investigation $[11,12]$.

The main objective of the study was to determine spatial variation and socio-economic determinants of $P$. falciparum infection in Korogwe district northeast Tanzania. Data from cross-sectional studies conducted between November 2005 and June 2007 in 14 villages of Korogwe district were used.

\section{Materials and methods Study area}

The study was conducted in 14 villages in Kwagunda, Msambiazi and Vugiri wards of Korogwe district in Tanzania (Figure 1) and individuals aged 0-19 years were involved. Selection of this age group was based on their variation in malaria immunity status [13]. The villages were chosen to represent areas with different malaria transmission intensities in preparation for sites for malaria vaccine trials. In this area, the major determinants of malaria transmission are altitude $[3,14]$ and level of urbanization.

Since January 2006, this area has been under Demographic surveillance system (DSS), which is updated after every four months. A baseline census survey was conducted in November 2005 where households and demographic information for all members were registered. About $15 \%$ of the population in the study area were children aged below five years while $37 \%$ were individuals aged 5-19 years. Three of the study villages are located in Korogwe township (Mtonga, Masuguru and Kwasemangube) and the rest are in rural areas. The altitude of the area ranges from 300 to $1000 \mathrm{mASL}$, (Figure 1) where four villages (Kwamasimba, Magundi, Kwamhanya and Vugiri) are located in the highlands ( $>500 \mathrm{mASL}$ ). Six villages (Mkokola, Mng'aza, Kwashemshi, Kwamasimba, Magundi and Kwamhanya) have passive case detection (PCD) of febrile episodes where community health workers treats persons presenting with malaria symptoms by using first line anti-malaria drugs. These villages were selected in order to collect longitudinal data of febrile cases. Among the selection criteria was different altitude which is a proxy for transmission intensity in this area [15].

\section{Data collection}

Four cross sectional malaria surveys were conducted in Nov-Dec 2005, May-Jun 2006, Nov-Dec 2006 and MayJun 2007. In the study area, the period between May and June is characterized with high malaria transmission

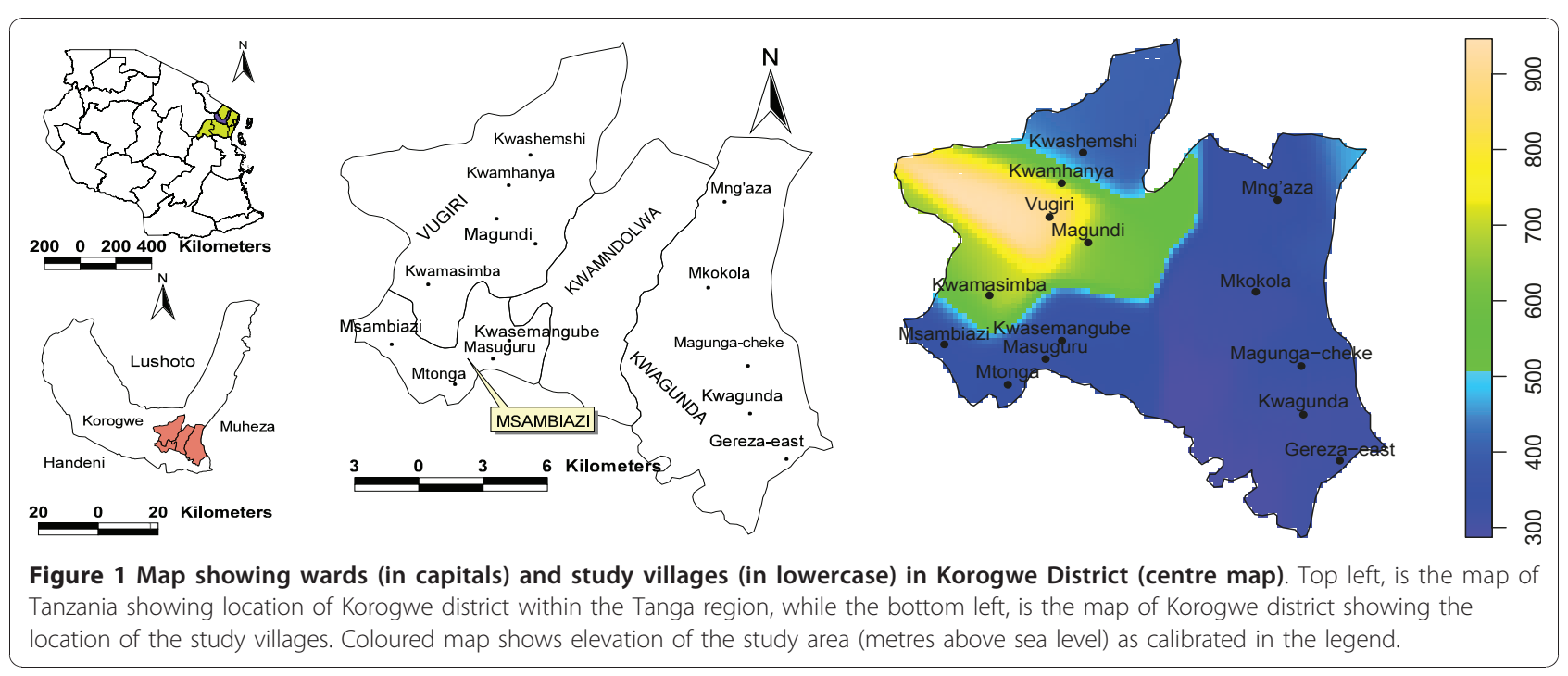


while Nov-Dec is a short transmission season. These periods correspond to the long (Mar-Jun) and short (Oct-Dec) rainy season. However, in the recent past there has been changes in the rainfall pattern.

About $40 \%$ of the children below five years of age and $15 \%$ of those aged 5-19 years were randomly selected from census database and adult individuals and parents/gurdians (for children) were sensitized to participate in the surveys. At each survey, demographic information of study participants were validated before physical examination and blood sampling. In brief, thick and thin smears were prepared from venous or finger prick blood, stained using Giemsa stain and read under a high power light microscope. For positive blood smears, parasites were counted against 200 white blood cells (WBCs) and a smear was declared negative after examining 100 high power fields. Household social economic data which included household properties, occupation of head of household and material used in construction of the house were collected during the first round of DSS (February-April 2006) using a questionnaire. Number of bed nets available and used in each household were collected during the first round of DSS, while information on whether an individual was using a bed net was sought during malaria cross-sectional surveys.

Mapping of households was done after the baseline census survey. Households positions ( $x$, y coordinates) were captured using a hand-held global positioning system (GPS) receiver and were downloaded into a computer and transferred to ArcView 3.2 software. Distance between points (households) were converted from degrees into meters by multiplying longitude and latitude by 111,000 assuming a radial distance of the equator [16]. A map of the study area was prepared by overlaying the village coordinates in the map of the wards in ArcView. GIS data were then linked to malariometric and socio-economic data and transferred to the $\mathrm{R}$ statistical software http://cran.r-project.org for detailed analysis.

Ethical clearance was granted by the Medical Research Coordinating Committee of the National Institute for Medical Research, Tanzania. Informed consent was obtained during the survey, both orally and in writing from adult individuals while for children it was obtained from their parents or guardians.

\section{Statistical analysis}

\section{Scoring of socio-economic status}

Principal component analysis was used to estimate the scores for the socio-economic status of the households. Some of variable which were considered in the principal component analysis were: source of power for lighting and cooking, ownership of radio, bicycle and mobile phone, ratio of number of sleeping bedrooms to number of household dwellers, occupation of head of household as well as number of animals and size of cultivated land owned by the family, see Table 1 . Number of bed nets per household size and type of wall and roof of house were included as risk factors in modeling risk of $P$. falciparum infection. These variables were selected as a proxy for socio-economic status because is difficult to obtain household income information [17]. The SES scores were categorized into three groups (low, medium and high SES) at the ratio of 40:40:20 for easy interpretation of results $[18,19]$.

Table 1 Variables used in assessing the socio-economic status (SES), their coding and estimated scores

\begin{tabular}{|c|c|c|}
\hline Variable & Variable type and code & SES score \\
\hline Source of power for lighting & Binary: ( $0=$ Local lamp[koroboi], 1 = kerosene lump/electricity) & 0.447 \\
\hline Source of power for cooking & Binary: (0 = Firewood, 1 = charcoal/kerosene/electricity) & 0.402 \\
\hline Possess Mobile phone & Binary: $(0=$ No, $1=$ yes $)$ & 0.390 \\
\hline Material used in making toilet walls & Binary: ( $0=$ Thatch/mud walls, $1=$ Bricks) & 0.365 \\
\hline Ownership of bicycle & Binary: $(0=$ No, $1=$ yes $)$ & 0.258 \\
\hline Occupation-formal employment & Binary: $(0=$ No, 1 = yes $)$ & 0.252 \\
\hline Source of water for domestic use & Ordinal: $(1=$ River, $2=$ Well, $3=$ Tap) & 0.223 \\
\hline Availability of radio & Binary: $(0=$ No, 1 = yes $)$ & 0.210 \\
\hline Occupation - Small business & Binary: $(0=$ No, 1 = yes $)$ & 0.147 \\
\hline Number of sleeping rooms/Household size & Continuous $[0-1][$ & 0.131 \\
\hline Ownership of house & Binary: (0 = No, 1 = yes) & 0.127 \\
\hline Occupation - Petty business & Binary: (0 = No, 1 = yes) & 0.092 \\
\hline Other occupation (such as masonry etc) & Binary: $(0=$ No, 1 = yes $)$ & 0.061 \\
\hline Number of cattle & Ordinal: $(0=$ none, $1=1-4,2=$ More than 4$)$ & 0.053 \\
\hline Number of goats/sheep & Ordinal: $(0=$ none, $1=1-10,2=$ More than 10$)$ & -0.021 \\
\hline Number of chicken & Ordinal: $(0=$ None, $1=1-20,2=$ More than 20) & -0.025 \\
\hline Occupation - Peasant farmer & Binary: $(0=$ No, 1 = yes) & -0.226 \\
\hline
\end{tabular}




\section{Modelling spatial variation of $\mathrm{P}$. falciparum infection}

In modeling spatial variation of $P$. falciparum infection, individuals who were found with P. falciparum parasite during the survey were considered as cases and those who were negative as multiple controls. Records of individuals were modeled as correlated observations since more than $50 \%$ of individuals had two or more observations in the four surveys. Let $\pi_{i j}$ be a probability of observing $Y_{i j}=1$ (P. falciparum case) in a person $i=1$ $\ldots, n$ seen in round $j=1 \ldots, J$ and living at location $x_{i} \in$ $R^{2}$; and let $Z_{i j}$ be a vector of length $p$ of associated explanatory variables. Using marginal model, this was modeled as a binomial random variable:

$$
\operatorname{logit}\left(\pi_{i j}\right)=\beta Z_{i j}+S\left(x_{i}\right),
$$

where $\beta$ is a vector of $p$ unknown regression parameters and $S\left(x_{i}\right): x_{i} \in R^{2}$ is a smooth function for residence locations; which estimates the spatial effect across the study area. Non-parametric method was used in modeling the spatial effect as described by Kelsall and Diggle [20], modified to take into account the repeated observations within individuals. Generalized estimating equation (GEE) [21] was used in modeling the risk of malaria. The GEE is a solution to the score equation:

$$
S_{\beta}(\beta, \alpha)=\sum_{i=1}^{n}\left(\frac{\partial \pi_{i j}}{\partial \beta}\right)^{\prime} \operatorname{Var}\left(Y_{i j}\right)^{-1}\left(Y_{i j}-\pi_{i j}\right)=0
$$

where, $\operatorname{Var}\left(Y_{i j}\right)=\operatorname{Var}\left(Y_{i j}, \beta, \alpha\right)$, and $\alpha$ is the correlation between $Y_{i j}$ and $Y_{i k}$. Estimation of $\alpha$ can be done by adding a second set of estimation equations $S_{\alpha}(\beta, \alpha)=0$ and solve the two equations simultaneously as detailed in Diggle et al [22]. Estimation of the odds of malaria was done in two parts: first by estimating the spatial component and then inserting the estimated spatial component into a model with other covariates and maximize iteratively. This was done by a back fitting procedure, where the spatial effect was estimated using gaussian kernel method (non-parametric). This was then inserted into GEE model using independent weights to obtain estimates of the parameters. The process of estimating parameters and spatial effect was done as follows:

Initialize $S\left(x_{i}\right)=0$, and estimate $\beta$ using GEE

Step 1: set the link function $\eta_{i j}=z_{i j}^{\prime} \hat{\beta}+\hat{S}_{i j}(x)$

- Construct an adjusted dependent variable $\mu_{i j}=\hat{\eta}_{i j}+\frac{y_{i j}-\hat{\pi}_{i j}}{\hat{\pi}_{i j}\left(1-\hat{\pi}_{i j}\right)}$ and weight $w_{i j}=\hat{\eta}_{i j}\left(1-\hat{\eta}_{i j}\right)$

Step 2: Fit an additive model $U=Z^{\prime} \hat{\beta}+\hat{S}(x)$ using a kernel regression with weights $w_{i j}$ as follows:
- set $r_{i j}=\mu_{i j}-z_{i j}^{\prime} \hat{\beta}$

- perform a weighted kernel regression using weights $w_{i j}$ and estimate the spatial effect as: $\hat{S}\left(x_{i}\right)=\frac{\sum w_{i j} K_{h}\left(x-x_{i}\right) r_{i j}}{\sum w_{i j} K_{h}\left(x-x_{i}\right)}$, where $K_{h}(u)=\frac{1}{h^{2}} K\left(\frac{u}{h}\right)$, is a spatial 2-dimensional kernel function and $h$ is a bandwidth parameter.

- regress $u_{i j}-\hat{S}\left(x_{i}\right)$ on $z_{i j}$ using weighted GEE to obtain new values of $\hat{\beta}$.

Repeated step 1 and 2 until the estimates converge.

Bandwidth parameter $h$ was estimated using cross validation technique which aims to find the values of $h$ which minimizes:

$$
C V(h)=\frac{1}{n} \sum w_{i j}\left[\mu_{i j}-\hat{s}^{-i}\left(x_{i}\right)\right],
$$

where $\hat{S}^{-i}\left(x_{i}\right)$ is the estimate of $S^{-i}\left(x_{i}\right)$ constructed with bandwidth $h$ using all except the pair $\left(x_{i}, \mu_{i j}\right)$ [20].

To predict the risk of malaria in un-sampled locations, a set of new locations were generated at regular interval (nine locations at each of one by one squared kilometres), and then the spatial effect was estimated at each new location using the kernel method as described above. Spatial risk maps corrected for edge effect were generated using spatstat [23], effect of covariates were fitted by geepack and yags package was used to estimate the pseudo-AIC of different models. These packages are contained in R statistical software. Model with the lowest pseudo-AIC was selected as the best model. A gaussian kernel and a bandwidth parameter of $1,000 \mathrm{~m}$ estimated as detailed above, were used in the entire analysis. To assess the spatial uncertainty, parametric bootstrap sampling was used whereby 200 datasets of similar size to the original dataset were generated by random sampling of individuals' records with replacement and then fitting the model as described above. This gave 200 maps and these were used to generate $2.5 \%$ and $97.5 \%$ percentile maps.

\section{Results}

A total of 12,298 data records of individuals from 14 villages collected during the four cross-sectional surveys were analyzed. Table 1 shows characteristic variables which were used to derive the socio-economic status of the households in the study area. Type of energy (for lighting and cooking) and possession of mobile phones were among the predictors of higher SES, while households headed by a peasant farmer were associated with lower SES. Individuals living in urban areas had higher SES than those living in highlands and rural lowlands. 
The mean scores of SES were 1.108 (95\%CI: 1.046 $1.169)$ in urban, -0.337 (95\% CI: $-0.369--0.304)$ in rural lowlands and -0.975 (95\%CI: -1.013 - -0.935) in highlands. The pooled P. falciparum prevalence was 35.9 (95\%CI: 34.9 - 36.9) in rural lowlands, 10.5 (95\%CI: 9.3 11.7) in urban and 11.2 (95\%CI: 9.7 - 12.7) in highlands.

\section{Spatial distribution of risk of P. falciparum infection}

Figure 2 shows distribution of $P$. falciparum parasite prevalence range during the four surveys and distribution of un-adjusted spatial log odds ratio for P. falciparum infection in the study area. The five villages on the right side of the map had higher malaria prevalence during the four surveys than the rest of the villages. A similar high risk surface in these villages is shown by un-adjusted smooth map, where north-eastern (Mng'aza) village and southeastern (Gereza-east) village had log-odds ratio exceeding one when compared to the average map (i.e log odds ratio of zero). Villages in the urban and highlands had similar low risk of $P$. falciparum infection.

When a model adjusted for risk factors (excluding altitude) was fitted, the maps showed a slightly different pattern of variation of risk of $P$. falciparum infection compared to un-adjusted map; for instance, the area with risk below log one increased to cover the three villages in the urban and two of the highland area, (Figure 3 , left).
Results from a model fitted with all risk factors including altitude, showed a shift in the risk of $P$. falciparum infection, whereby the urban area remained with low risk of infection, while the areas surrounding the urban were at a moderate risk. Villages which had highest risk remained the same, however, a village situated at the highest altitude (Vugiri) had significantly higher risk compared to the average map, (Figure 3, right). Maps of 2.5th and 97.5th percentiles from model fitted with all risk factors including altitude (Figure 4) showed that the estimates of spatial effects fitted in Figure 3 (right) were consistent. The pseudo-AIC statistics showed that model with all covariates including altitude had lowest value $(27,753.4)$ while one without covariate had the highest value $(28,184.7)$, indicating that the full model was the best.

\section{Risk factors of P. falciparum infection}

Table 2 shows results for variables that were associated with $P$. falciparum infection in the study area as fitted by a regression model outlined above. Individuals who were using bed nets had a 20.4\% (95\%CI: 11.7 - 28.4; p $<0.001)$ lower risk of infection than those not using nets. Compared to individuals living in households without bed nets and independent of current use of bed nets; individuals who were living in households where the ratio of bed nets to people was $(0-0.4]$ (i.e a ratio

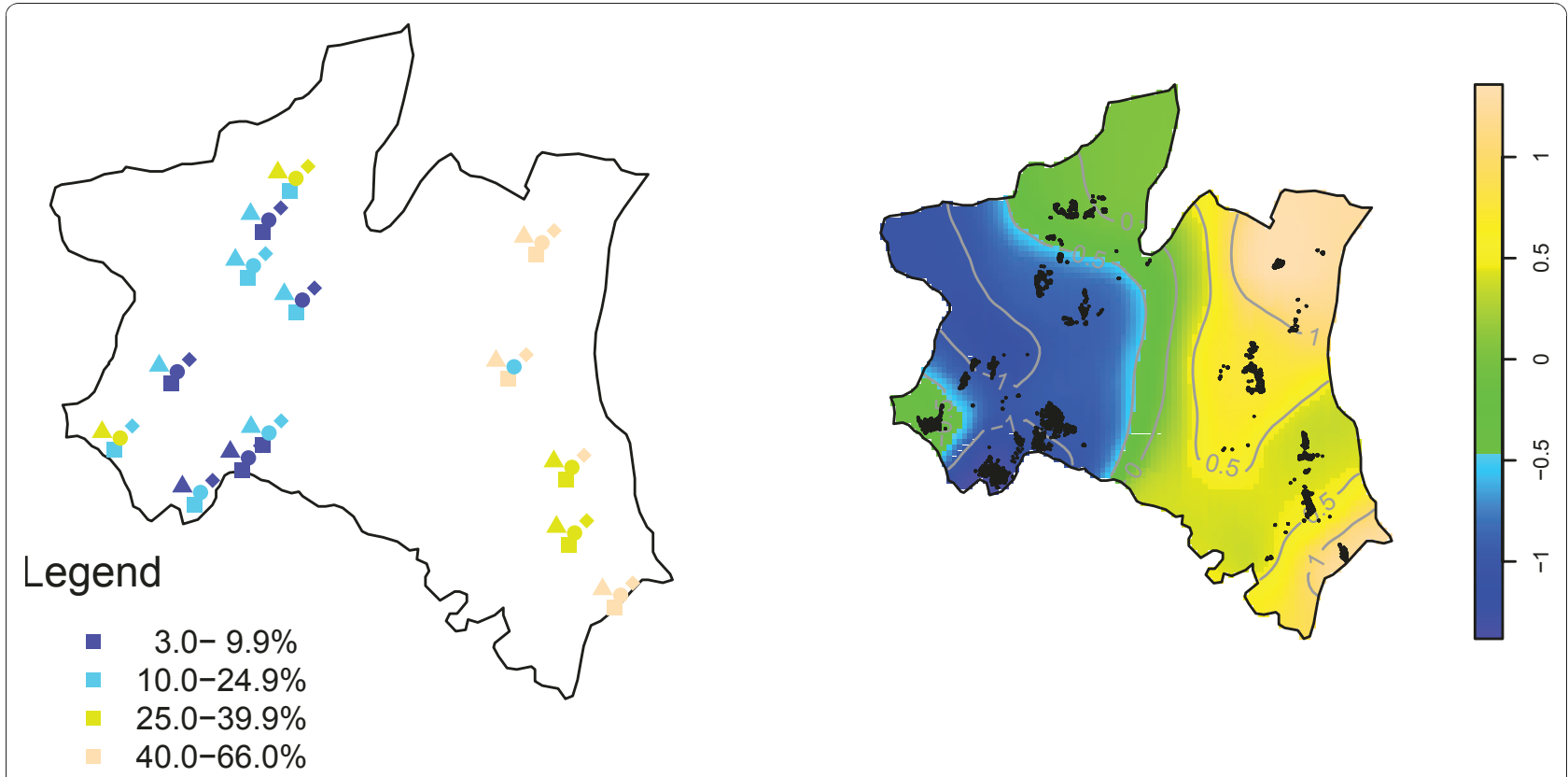

Figure 2 Distribution of ranges of parasite prevalence (left) and smoothed un-adjusted risk of P. falciparum (right) during 2005-2007 cross-sectional surveys. In the left, colours represents ranges of parasite prevalence as shown in the legend, while symbols shows survey 1-4 indicated by square, cycle, triangle and diamond, respectively. Smooth colors shows log odds ratio of $P$. falciparum infection, where deep blue and light brown show areas with lowest and highest risks, respectively. Point marks (right) shows residential location of individuals who were sampled during Nov 2005-May 2007 cross sectional surveys 


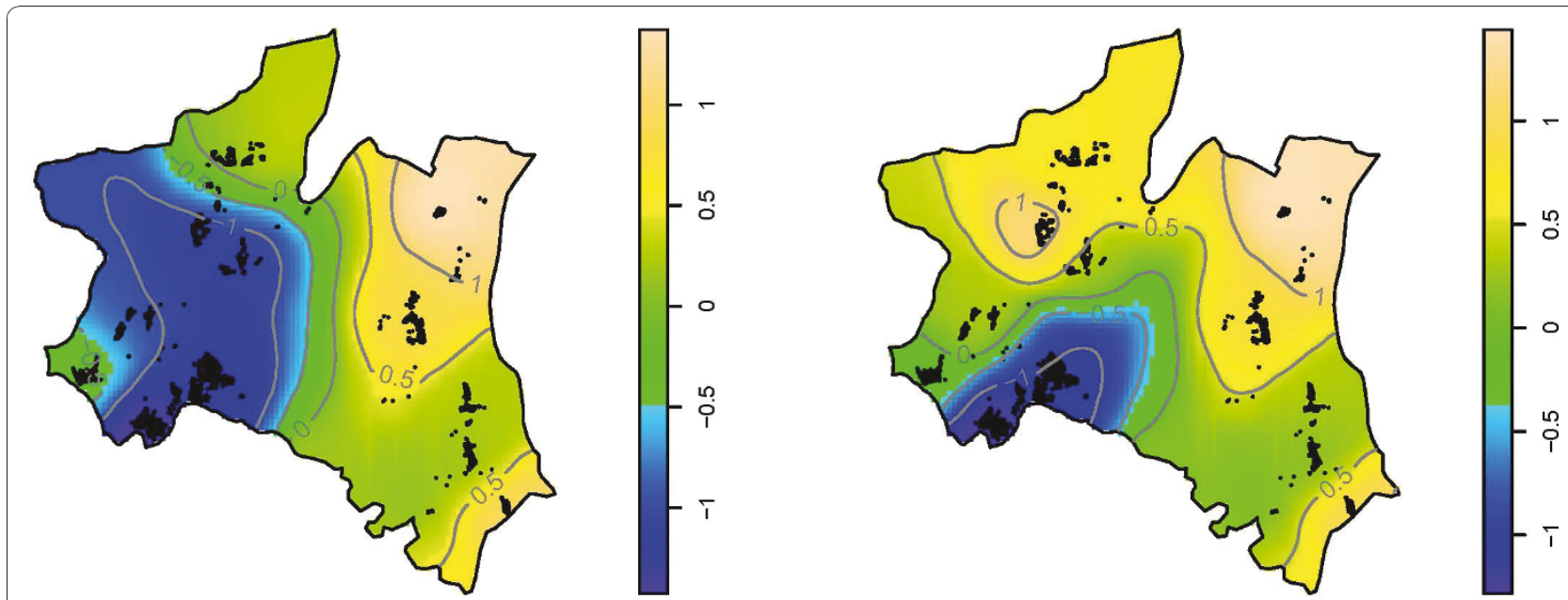

Figure 3 Map showing the spatial variation in risk of $P$. falciparum infection in the study area. Prediction based on model without altitude (left) and with altitude (right). Smooth colors shows log odds ratio of P. falciparum infection, where deep blue and light brown show areas with lowest and highest risks, respectively. Point marks shows residential location of individuals who were sampled during Nov 2005-May 2007 cross sectional surveys.

greater than zero and $\leq 0.4)$, the risk of $P$. falciparum infection were reduced by $19.1 \%$ (95\% CI: 8.9-28.2, p < 0.001 ) while in households with a ratio above 0.4 , the risk was reduced by $39.3 \%$ (95\%CI: $28.9-48.2, \mathrm{p}<0.001$ ).

Socio-economic status of household was also a significant predictor of malaria infection, where the risk was 60.5\% higher $(95 \% \mathrm{CI}: 33.6-92.7, \mathrm{p}<0.001)$ in households with moderate and $61.1 \%$ higher $(95 \% \mathrm{CI}$ : 35.9 91.0, $\mathrm{p}<0.001$ ) in low SES compared to those with highest SES. Moreover, individuals living in houses built of mud walls had a 15.5\% (95\%CI:00.1 - 33.3, p < 0.048) higher risk compared to those living in houses built of bricks, while those who were living in houses with thatched roof were at a $17.3 \%(95 \% \mathrm{CI}: 4.1-32.2$, p < 0.009 ) higher risk than those with houses roofed by iron sheets. Individuals living in villages with passive case detection were also at a reduced risk of $P$. falciparum infection by $24.0 \%$ (95\%CI: $14.9-32.0, \mathrm{p}<0.001)$, Table 2.

\section{Discussion}

The aim of this study was to determine the spatial variation and socio-economic determinants of P. falciparum infection in an area with different transmission intensities in northeastern Tanzania. Results from this study showed high spatial variability in the risk of $P$.

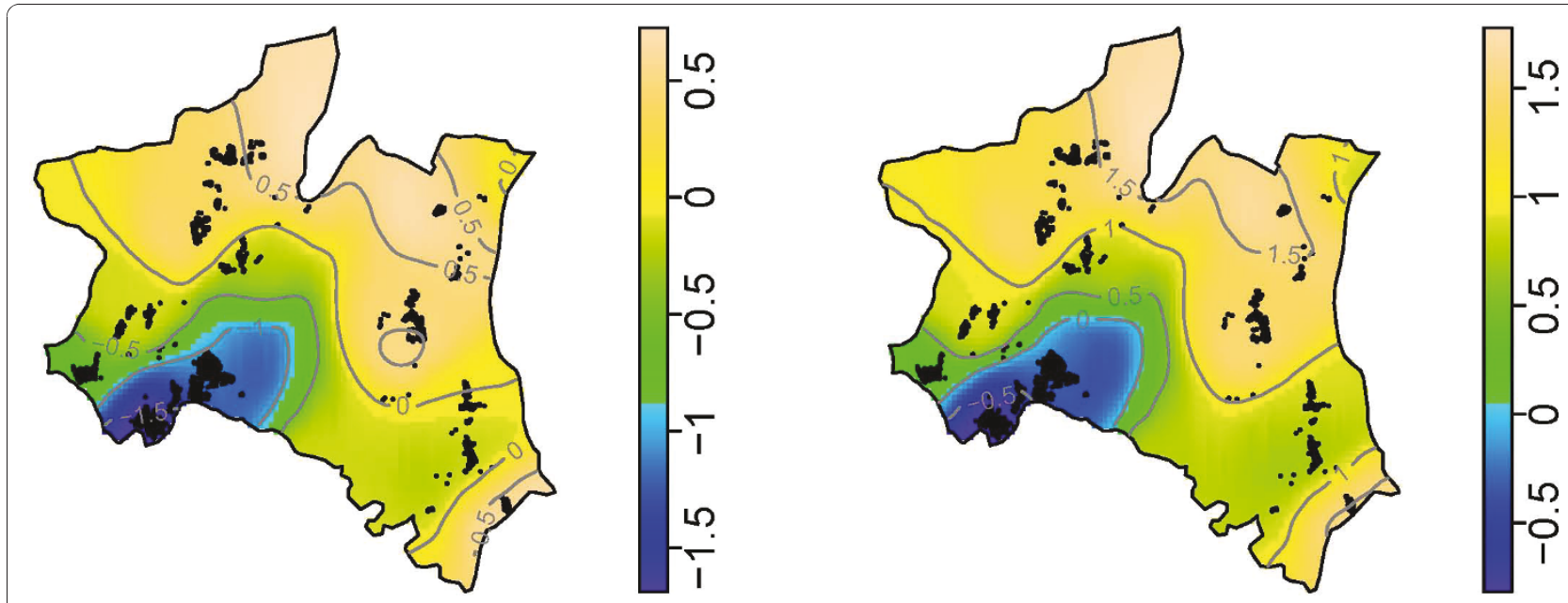

Figure 4 Map showing 2.5\% (left) and $97.5 \%$ (right) percentiles of risk of P. falciparum infection predicted from 200 bootstrap sampling adjusted for risk factors and altitude. Smooth colors shows log odds ratio of $P$. falciparum infections, where deep blue and light brown show areas with lowest and highest risks, respectively. 
Table 2 Distribution of multiple regression parameter estimates for factors associated with risk of $P$. falciparum infection as estimated by equation (1), where the spatial component is presented in Figure $(3$, right)

\begin{tabular}{|c|c|c|c|c|}
\hline Variable & OR & $95 \%$ & $\mathrm{Cl}$ & P-value \\
\hline Age & 1.41 & 1.345 & 1.477 & $<0.001$ \\
\hline Age squared & 0.983 & 0.980 & 0.985 & $<0.001$ \\
\hline bed net use & 0.796 & 0.716 & 0.883 & $<0.001$ \\
\hline Bed net rate (Zero) & 1 & & & \\
\hline Bed net rate $(0-0.4]$ & 0.809 & 0.718 & 0.911 & $<0.001$ \\
\hline Bed net rate (0.4-1] & 0.607 & 0.518 & 0.711 & $<0.001$ \\
\hline SES -(High) & 1 & & & \\
\hline Medium & 1.605 & 1.336 & 1.927 & $<0.001$ \\
\hline Low & 1.611 & 1.359 & 1.910 & $<0.001$ \\
\hline Type of roof (Thatch) & 1.173 & 1.041 & 1.322 & 0.009 \\
\hline Type of wall (Mud walls) & 1.155 & 1.001 & 1.333 & 0.048 \\
\hline Year -2005 & 1 & & & \\
\hline 2006 & 0.853 & 0.747 & 0.974 & 0.019 \\
\hline 2007 & 0.778 & 0.640 & 0.946 & 0.012 \\
\hline Short rains & 1.124 & 0.995 & 1.269 & 0.059 \\
\hline PCD & 0.760 & 0.680 & 0.851 & $<0.001$ \\
\hline Altitude (by 100m) & 0.664 & 0.640 & 0.689 & $<0.001$ \\
\hline
\end{tabular}

$\mathrm{PCD}=$ Precense of passive case detection, $\mathrm{SES}=$ Socio-economic status

falciparum infection. The highest risk was found in the lowland rural area, while the lowest risk was in the urban area $[24,25]$. The pattern of spatial variation in risk of $P$. falciparum infection were shown to vary in the maps adjusted for risk factors. A map adjusted for risk factors excluding altitude indicated a similar risk between the highlands and urban areas. This map had lower spatial heterogeneity in risk of P. falciparum infection than un-adjusted map. This shows that some of the variations which were seen in the un-adjusted map were due to risk factors included in the model.

The risk in highland area was shown to increase substantially when the effect of altitude which is a proxy for malaria transmission in this area was included in the model [15]. Inhabitants of one of the villages in the highland (Vugiri) were shown to have a risk almost similar to that of inhabitants of the lowland village (Mng'aza). Inhabitants of Vugiri village did not have passive case detection system (PCD); however, since the map was also adjusted for the effect of PCD, the risk would be expected to be similar to that of surrounding villages. This indicates that the transmission in this village could not be explained by variables included in the model.

The reason for high risk in Mng'aza and Mkokola villages which are located in the lowland area could be proximity to Lwengera River which flows on the eastern side of the two villages, while a stream which flows along the south-east border of Gereza-east village might be the reason for high infection in this village. These water bodies might be the source of the breeding sites for mosquitoes and hence increased transmission.

It is obvious that some of important risk factors such as use of mosquito repellents, management of surroundings, and distribution and distances to mosquitoes breeding sites were not accounted for in these models. Inclusion of these information could explain more of the observed spatial variation in the study area. Unfortunately, no recent entomological studies were done in the study area which could provide more information on mosquito breeding and transmission dynamics.

Socio-economic status of households was an important risk factor for malaria in the study area, where households of lower to moderate socio-economic status were at a more than $60 \%$ higher risk compared to households of highest socio-economic status. Similar findings have been reported elsewhere [6,26,27]. Poor housing was also among the risk factors for malaria in the study area, whereby individuals living in houses built of mud and thatched roofs were at a higher risk compared to individuals of similar socio-economic status but living in better houses. Similar findings were reported by a study in Sri Lanka where mosquito abundance was associated with the poor housing construction [28] and in South Africa where a case-control study showed a six fold risk of malaria in houses built of mud walls compared to bricks [27]. This indicates that better houses do not give easy entry and hiding places for mosquitoes.

High coverage of bed nets was an important finding in our study area; this was associated with reduction of infection regardless of whether an individual was using a bed net. For example, individual living in a household with five persons and two bed nets was at $20 \%$ lower risk of infection than those living in households without a net. This indicates the importance of high coverage of bed nets in reduction of malaria infection [29-31]. A study done in malaria endemic area of Tanzania [32], showed that the density of bed nets coverage in households was the only significant covariate associated with reduction in mortality, which is an important indicator in reduction of malaria burden.

\section{Conclusion}

Results from this study showed high variability of the risk of malaria within the study area. Altitude, SES, high bed net coverage and urbanization are amongst the factors associated with the spatial variability in malaria. Improving access to means of protection against mosquitoes and living standards of the population might have a significant impact on the burden of malaria. The risk maps helped to identify areas with high risk of $P$. falciparum infection and this can be useful in malaria 
control in the area. Prediction maps outlined in this study can be used in priority settings; whereby the maps un-adjusted for altitude can be used to show areas with high risk where treatment should be diverted to, while maps adjusted for risk factors indicates areas where transmission is higher than would be expected and prevention might be the most effective. These map could also be used to identify areas where further investigation is needed to ascertain the cause of high risk of $P$. falciparum infection.

\section{List of abbreviations}

ASL: Above sea level; DSS: Demographic surveillance system; GEE: Generalized estimating equation; GIS: Geographical information system; GPS: Global positioning system; OR: Odds ratio; PC: Principal component; PCA: Principal component analysis; PCD: Passive case detection; SES: Socioeconomic status; WBC: White blood cells.

\section{Acknowledgements}

We thank all individuals who agreed to participate in the study and the villages' leaders for their cooperation. Korogwe district authorities are thanked for good cooperation and NIMR Director General for giving permission to publish. We thank the teams from NIMR Tanga Centre and ENRECA project in Korogwe for their support during data collection while Aza Kimambo is acknowledged for collecting GIS data. The study was financed by a grant from the African Malaria Network Trust (AMANET) and DANIDA through ENRECA Project.

\section{Author details}

${ }^{1}$ National Institute for Medical Research, Tanga Centre, Tanzania. ${ }^{2}$ Centre for Medical Parasitology, Institute for Medical Microbiology and Immunology, University of Copenhagen, Denmark. ${ }^{3}$ Department of Biostatistics, University of Copenhagen, Denmark.

\section{Authors' contributions}

BPM participated in designing the study, data collection and analysis, and manuscript writing. MLK and DSI participated in designing the study and data collection. JPA and MML participated in designing the study, supervision of field and laboratory work. FF-Management of data. TGT participated in designing the study and scientific suggestions. THS Participated in analysis design and commented in all stages of statistical analysis. All authors read and approved the final manuscript for publication.

\section{Competing interests}

The authors declare that they have no competing interests.

Received: 23 February 2011 Accepted: 25 May 2011

Published: 25 May 2011

\section{References}

1. Kilama W: The $10 / 90$ gap in sub-Saharan Africa: resolving inequities in health research. Acta Trop 2009, 112(Suppl 1):S8-S15.

2. Ministry of Health: Plan of action for implementing Roll Back Malaria in Tanzania. Tech. rep., Ministry of Health; 2001.

3. Bodker R, Akida J, Shayo D, Kisinza W, Msangeni H, Pedersen E, Lindsay S: Relationship between altitude and intensity of malaria transmission in the Usambara Mountains, Tanzania. J Med Entomol 2003, 40:706-717.

4. Robert V, Macintyre K, Keating J, Trape J, Duchemin J, Warren M, JC B: Malaria transmission in urban sub-Saharan Africa. Am J Trop Med Hyg 2003, 68:169-176.

5. Kahigwa E, Schellenberg D, Sanz S, Aponte J, Wigayi J, Mshinda H, Alonso P, Menendez C: Risk factors for presentation to hospital with severe anaemia in Tanzanian children: a case-control study. Trop Med Int Health 2002, 7:823-830.

6. Biritwum R, Welbeck J, Barnish G: Incidence and management of malaria in two communities of different socio-economic level, in Accra, Ghana. Ann Trop Med Parasitol 2000, 94:771-778.
7. Martens W: Climate change and malaria: exploring the risks. Med War 1995, 11:202-213.

8. Omumbo J, Hay S, Snow R, Tatem A, Rogers D: Modelling malaria risk in East Africa at high-spatial resolution. Trop Med Int Health 2005, 10:557-566.

9. Goovaerts P: Geostatistical analysis of disease data: accounting for spatial support and population density in the isopleth mapping of cancer mortality risk using area-to-point Poisson kriging. Int I Health Geogr 2006, 5:52.

10. Webster T, Vieira V, Weinberg J, Aschengrau A: Method for mapping population-based case-control studies: an application using generalized additive models. Int J Health Geogr 2006, 5:26.

11. Hay S, Guerra C, Gething P, Patil A, Tatem A, Noor A, Kabaria C, Manh B, Elyazar I, Brooker S, Smith D, RA M, RW S: A world malaria map: Plasmodium falciparum endemicity in 2007. PLoS Med 2009, 6:e1000048.

12. SL M: GIS and health care. Annu Rev Public Health 2003, 24:25-42.

13. Smith D, Guerra C, Snow R, Hay S: Standardizing estimates of the Plasmodium falciparum parasite rate. Malar I 2007, 6:131.

14. Lusingu J, Jensen A, Vestergaard L, Minja D, Dalgaard M, Gesase S, Mmbando B, Kitua A, Lemnge M, Cavanagh D, Hviid L, Theander T: Levels of plasma immunoglobulin $\mathrm{G}$ with specificity against the cysteine-rich interdomain regions of a semiconserved Plasmodium falciparum erythrocyte membrane protein 1, VAR4, predict protection against malarial anemia and febrile episodes. Infect immun 2006, 74:2687-2875.

15. Drakeley C, Carneiro I, Reyburn H, Malima R, Lusingu J, Cox J, Theander T, Nkya W, Lemnge M, Riley E: Altitude-dependent and -independent variations in Plasmodium falciparum prevalence in northeastern Tanzania. J Infect Dis 2005, 191:1589-1598.

16. Kirvan A: Latitude/Longitude, NCGIA Core Curriculum in GIScience, (retrieved September 2009).[http://www.ncgia.ucsb.edu/giscc/units/u014/ u014 f.html].

17. Moris SSaul: Measuring Health Equity in Small Areas: Findings from Demographic Suveillance Systems, Aldershot: Ashgate 2005 chap. Epidemiology and the Study of Socio-economic Inequities in Health 1-13.

18. Filmer D, Pritchett L: Estimating Wealth Effects Without Expenditure Data-Or Tears: An Application To Educational Enrollments In States Of India. Demography 2001, 38:115-132.

19. Vyas S, Kumaranayake L: Constructing socio-economic status indices: how to use principal components analysis. Health Policy Plan 2006, 21:459-468.

20. Kelsall J, Diggle P: Non-parametric estimation of spatial variation in relative risk. Stat Med 1995, 14:2335-2342.

21. Zeger $S$, Liang K: Longitudinal data analysis for discrete and continuous outcomes. Biometrics 1986, 42:121-130.

22. Diggle $\mathrm{P}$, Heagerty $\mathrm{P}$, Liang K, Zeger S: Analysis of Longitudinal Data. 2 edition. New York: Oxford University Press; 2002.

23. Baddeley $A, R T$ : spatstat: An R package for analyzing spatial point patterns. J Stat Softw 2005, 12:1-42

24. Mmbando B, Segeja M, Msangeni H, Sembuche S, Ishengoma D, Seth M, Francis F, Rutta A, Kamugisha M, Lemnge M: Epidemiology of malaria in an area prepared for clinical trials in Korogwe, north-eastern Tanzania. Malar J 2009, 8:165.

25. Hay S, Guerra C, Tatem A, Atkinson P, Snow R: Urbanization, malaria transmission and disease burden in Africa. Nat Rev Microbiol 2005, 3:81-90.

26. Silue K, Raso G, Yapi A, Vounatsou P, Tanner M, N'goran E, Utzinger J: Spatially-explicit risk profiling of Plasmodium falciparum infections at a small scale: a geostatistical modelling approach. Malar J 2008, 7:111.

27. Coleman M, Coleman M, Mabaso M, Mabuza A, Kok G, Coetzee M, Durrheim D: Household and microeconomic factors associated with malaria in Mpumalanga, South Africa. Trans R Soc Trop Med Hyg 2010, 104:143-147.

28. Konradsen F, Amerasinghe P, van der Hoek W, Amerasinghe F, Perera D, Piyaratne M: Strong association between house characteristics and malaria vectors in Sri Lanka. Am J Trop Med Hyg 2003, 68:177-181.

29. Maxwell C, Msuya E, Sudi M, Njunwa K, Carneiro I, Curtis C: Effect of community-wide use of insecticide-treated nets for 3-4 years on malarial morbidity in Tanzania. Trop Med Int Health 2002, 7:1003-1008.

30. Deressa W, Ali A, Berhane Y: Household and socioeconomic factors associated with childhood febrile illnesses and treatment seeking behaviour in an area of epidemic malaria in rural Ethiopia. Trans $R$ Soc Trop Med Hyg 2007, 101:939-947. 
31. Curtis C, Maxwell C, Lemnge M, Kilama W, Steketee R, Hawley W, Bergevin Y, Campbell C, Sachs J, Teklehaimanot A, Ochola S, Guyatt H, Snow R: Scaling-up coverage with insecticide-treated nets against malaria in Africa: who should pay. Lancet Infect Dis 2003, 3:304-307.

32. Gosoniu L, Vounatsou P, Tami A, Nathan R, Grundmann H, Lengeler C: Spatial effects of mosquito bednets on child mortality. BMC Public Health 2008, 8:356.

doi:10.1186/1475-2875-10-145

Cite this article as: Mmbando et al:: Spatial variation and socioeconomic determinants of Plasmodium falciparum infection in northeastern Tanzania. Malaria Journal 2011 10:145

Submit your next manuscript to BioMed Central and take full advantage of:

- Convenient online submission

- Thorough peer review

- No space constraints or color figure charges

- Immediate publication on acceptance

- Inclusion in PubMed, CAS, Scopus and Google Scholar

- Research which is freely available for redistribution

Submit your manuscript at www.biomedcentral.com/submit 